sary, for it has been thought, though this is disputed, that it stirs up a simple adenoma of the thyroid into such activity that it becomes a toxic adenoma or secondary exophthalmic goitre.

The difference between the treatment of deficient and excessive endocrine secretion is noteworthy; when deficient, as in myxœdema, the indication is simple, namely, to supply it; but when excessive or perverted, as in exophthalmic goitre, acromegaly, and the alteration of growth and of the secondary sexual characters seen in tumours of the adrenal cortex, medicinal treatment is not so effective ; it is true that in hyperthyroidism and exophthalmic goitre iodine exerts a beneficial influence, but in Graves' disease it is found to be most useful in preparing the patient for operation. With excessive secretion it is reasonable to give belladonna, but its effects are not constant, neither in the case of exophthalmic goitre are those of adrenalin; the extract of the adrenal cortex, though recommended, has not been widely tried. The destructive action of $\mathrm{X}$-rays has given good effects in many cases of Graves' disease, but it is attended by the disadvantage of producing adhesions which render operation more difficult. In acromegaly extracts of the glands alone, or combined with thyroid extract, have been much tried, but with little success, except perhaps in diminishing headache; removal of the adenomatous gland is a severe, but in expert hands a successful operation.

In the remarkable changes of growth and the secondary sexual characters seen in cases of adrenal cortical tumours no measure short of removal of the tumour, which has been done in a small number of cases with success, notably in those reported by Gordon Holmes, and quite recently by Murray and Simpson, is at present available.

\section{ETIOLOGY AND TREATMENT OF ECZEMA.}

A LECTURE DELIVERED BEFORE THE HARROGATE MEDICAL SOCIETY

(Under the auspices of the Fellowship of Medicine.)

By W. GRIFFITH,

M.B., M.R.C.P.,

Physician and Joint Chesterfield Lecturer in Dermatology at the St. John's Hospital for Diseases of the Skin.

IN the first place I would like to thank the Harrogate Medical Society for the honour they have done me by inviting me to address them.

The subject chosen for discussion is the "Etiology and Treatment of Eczema," a subject in which, as a practising dermatologist, I am especially interested.

Harrogate has a traditional and well deserved reputation for the treatment of eczema and other skin diseases.

Many of my eczema patients tell me they have been to Harrogate and that they have been benefited or cured, and I hope to hear to-night at first hand some details of the methods which are practised here with so much success.

I have not come to teach, but to learn.

Many years' experience in a busy metropolitan skin clinic has convinced me that the usual textbook descriptions of eczema are lacking in completeness ; they are based on the views of Hebra and the Vienna school of his day, which maintained that eczema was a condition due entirely to external irritants acting on the skin.

This gives no explanation of eczema due to a special susceptibility in certain individuals.

The action of an irritant on the skin of a normal individual does not produce the clinical manifestations which we call eczema.

If a simple irritant, say hot water as the most familiar, be applied to the skin of a normal individual, it causes pain; redness, 
and swelling; or blistering or ulceration according to the intensity of the heat; simple inflammatory reactions of various degrees.

The following points are worthy of note. It will produce the same reaction in all normal persons; the reaction will be directly proportional to the intensity of the heat and the duration of the exposure; it will be limited to the area of application and will cease when the irritant is removed; and it will be similar if the heat be reapplied on a different site; if a blister forms and it be pricked it empties and the discharge ceases.

\section{Cases.}

(I) A Case of Simple Dormalitis in a Paticnt of Normal Susceptibility.-The patient presented himself with an acute exuding dermatitis about the left elbow-joint. The skin of the rest of the body was unaffected. He stated that he had rubbed the skin with a liniment because of pain in the elbow.

The dermatitis was limited to the part rubbed, showed no sign of extension and was obviously due to the irritant. It quickly disappeared on the discontinuance of the liniment.

An attack of eczema is essentially different, a severe attack may occur from a very slight irritation, entirely out of proportion to the intensity of the irritant; the symptoms will only occur in certain individuals, they do not disappear on the removal of the irritant, but last indefinitely and relapse and recur on the slightest provocation ; they rapidly spread far beyond the area injured, and develop symmetrically on the opposite side of the body.

When the blisters of eczema are broken the discharge is prolonged and continuous.

An essential feature of eczema is the extraordinary susceptibility of the individual to irritants which are harmless to normal people; and this susceptibility may be congenital or acquired.

A few common examples of susceptibility illustrate this feature:-
Several people may handle a plant-the Primula obconica-and of these, perhaps two members of the same family may deve. lop severe attacks of eczema, the others of the party being unaffected: a condition of of familial congenital susceptibility.

A family may eat strawberries, and perhaps a father and daughter may have an attack of eczema, while the mother and sons eating the same batch of strawberries may be unaffected : a familial susceptibility to an article of diet.

A surgeon may use carbolic lotion to disinfect his hands and stand it well for years; he suddenly gets an attack of eczema, and has to avord carbolic acid for evermore. A pathologist may handle formaline, or a dentist local anæsthetics, and the same kind of thing happens, all examples of acquired susceptibility.

Such occurrences are common in trades such as baking, photography, processengraving, sugar-boiling, hair-dressing, gardening and many others.

Susceptibility to woollen garments, to hair dye, to dyed fur, as well as to certain articles of diet, such as milk, eggs, shellfish and strawberries, is also common.

Attacks and relapses of eczema are frequent after exposure to causes of this kind and prove the existence of an inherent or acquired susceptibility.

It is to be noted that though the irritants may be various and may be applied externally or taken internally, or possibly absorbed from some septic focus, the reaction of the skin in these persons is always the same, an attack of eczema.

It is also well known that these same substances are common causes of asthma, urticaria, and angioneurotic cedema, and that the association of eczema and asthma is a trequent one.

(2) A Case of Relapsing Eczenna in a Sensitized Subject.-A clergyman who had retired from active duties because of attacks of asthma. His father and nephew were also subject to asthma. He wore flannel 
shirts because of his chest condition, and ate eggs for breakfast every morning. $\mathrm{He}$ knew of no cause for his eczema, and there was no history of any external irritant. He had suffered for some months from severe relapsing attacks of generalized eczema in spite of all precautions and treatment by specialists. He was advised to wear silk instead of wool, to avoid eggs, and to go to Harrogate for a course of detoxication.

The clinical signs of eczema are too familiar to require detailed description; a sudden burst of erythema and œdema, spreading symmetrically over a fairly well defined vasomotor area, the face, neck and arms, or the genitals and pelvis being common sites.

The exciting cause is often a mystery.

The erythema goes on to vesiculation and exudation, a copious continuous discharge ensues, and the progress is one of frequent relapses for weeks, months or years.

The histological picture differs in no respect from that of a simple inflammation of the skin, vascular dilatation of the papillary region, œdema of the epidermis, dilatation of the intercellular spaces, and the formation of vesicles on the skin surface.

When the attack ceases the skin quickly returns to its normal condition.

It is not a change in the structure of the skin, but simply a vascular reaction, not very different except in intensity to what normally occurs during sweating.

What is the explanation of these phenomena? The view that I submit to you is that eczema is not a structural disease of the skin, but simply a vascular reaction on the part of the organism to get rid of a poison, and that the susceptibility and the attacks are salutary, protective and eliminative.

The process consists of dilatation of the skin capillaries and arterioles, by which a large volume of blood is brought near to the external surface, the fluid portion of the blood exudes out of the vessels and finds an outlet through the skin, and the continuous discharge carries away the poison with it.
A study of the immediate cause of the vascular dilatation is an interesting one.

Some researches carried out by Sir Thomas Lewis give an explanation [I].

Lewis's view is that when the cells of the skin are damaged, they liberate a substance which acts as a vaso-dilator, the substance having many of the properties of histamine.

Lewis shows that this substance acts in three ways: it causes dilatation of the capillaries, it increases their permeability, and reflexly causes dilatation of the arterioles of the area.

These phenomena resemble the vascular changes which occur in anaphylaxis.

It has been demonstrated experimentally that in anaphylaxis there occurs before the vascular dilatation a vascular contraction-a squeezing out of the blood from the arterioles into the capillaries-due to the action of the anaphylactic substance on the unstriped muscles of the vessels. It is this same action on unstriped muscle that causes the broncho-spasm of asthma. In some individuals the bronchial effect predominates, in others the skin reaction. Clinically a severe fulminating attack of eczema, occurring from a slight or an unknown cause, suggests at once that the reaction is the rousing up of some latent susceptibility of which dilatation of the cutaneous vessels is the basic feature.

The same kind of vaso-dilatation occurs in surgical and histamine shocks and in anaphylaxis.

In experimental shocks the withdrawal of blood from the main vessels to the skin capillaries is sufficient to cause failure of the circulation, collapse and death, the subject bleeds into its own capillaries.

In eczema the cutaneous dilatation is sufficient to bring a large volume of blood to the skin surface without unduly interfering with normal circulation.

It is on these grounds that I submit that eczema is not a disease of the skin, but an eliminative process.

The skin is one of the important 
eliminating organs of the body, excreting normally about as much fluid as the kidneys, and when the kidneys are defective it can carry on a large part of their function, urea being freely eliminated by the skin in these conditions. When the organism is hampered by toxins which the kidneys cannot cope with, and which normal sweating cannot get rid of, this process of eczema comes into play and excretes the poison in its discharge.

Certain conditions are well known to predispose to eczema ; they are dietary conditions which lead to toxæmia and defective action of the eliminating organs of the body.

Toxæmia is probably a predisposing cause of the susceptibility to the irritants I have mentioned.

The most frequent food poisons affecting these cases are milk, eggs, shell-fish, strawberries, pork, and excess of starchy or fatty food.

Infantile eczema is common during the period of first dentition; this is also the time when the child is weaned and errors of dieting are prevalent. Bottle-fed babes and rickety children are notoriously subject to eczema.

Thin chronic dyspeptics and stout overfed subjects are also susceptible, so are persons suffering from Bright's disease, diabetes and constipation, and women at the catamenia and the menopause.

Defective eliminative function of the skin itself is also a common cause of eczema.

Ichthyotic subjects who do not sweat, and individuals who are defective in general sweating, but sweat excessively on the hands and feet, are frequently eczematous.

The conclusion I wish to draw from these considerations is that eczema and toxæmia are closely associated.

This conception of eczema gives us two main indications for treatment :-

(1) Avoidance of the substance to which the patient is susceptible; and,

(2) The removal of the susceptibility by ridding the patient of the toxæmia, and the restoration of normal metabolism and the normal functioning of the eliminative organs.

We have to bear in mind that though a patient in this toxic state may have a special intolerance to some specific substance, there always exists in these cases a general susceptibility; for instance, a patient sensitized to dyed fur (paraphenylendiamin) may relapse at once on exposure to bright sunlight; or by an indiscretion in diet, or on a visit to the seaside.

\section{TREATMENT.}

During an acute attack there will be an inflamed skin, severe itching, a copious discharge, and a good deal of nervous restlessness, and probably constipation to deal with.

The best way is to encourage elimination rather than to attempt to tinker with the symptoms. Free action of the bowels is imperative, and a dose of calomel with pulv. jalapæ co. followed by a saline serves the purpose well. Copious draughts of warm fluid will serve to flush the intestines, the skin and the kidneys. Fasting or a starvation diet will take the strain off the eliminative organs, and sedatives can be given if the irritation or insomnia is extreme. Strong astringent lotions to check the discharge from the skin, or local or constitutional steps to cause vaso constriction, will probably do more harm than good, and add to the patient's discomfort, while warm boracic fomentations give comfort and ease.

The acute attacks pass off, it is the relapses and recurrences which chiefly perturb the patient and the physician; these may occur at all sorts of intervals and continue for weeks, months, or years.

The first thing to do, if the cause is obvious, is to remove the patient from the influence which specially affects him.

Industrial workers will require a long rest from their trade, and poisonous plants, hair 
dye, and shell fish must be avoided by those susceptible to them.

The patient will probably know by bitter experience what articles of diet to avoid.

In cases where the cause is obscure a thorough search must be made for it. Sometimes the poison may come from a septic focus in the mouth, nasal cavity, gallbladder or appendix. The digestion may be at fault and ill-digested material may gain entrance to the blood-stream. If the diet is suspected the patient may be kept experimentally on a diet from which certain classes of food are excluded ; milk, eggs, fish or fruit may be withheld and the result watched, or the cutaneous food tests may give a clue.

The emunctories must be examined as a matter of course, the urine tested for albumin, sugar, and for indications of acidæmia or intestinal toxins. Absorption of toxins from the intestines is probably a common cause, and this is often due to some organic disease such as chronic ulcers.

Any defective conditions of this kind must, if possible, be remedied and steps taken to relieve the patient of his toxæmia. To this end the following methods are the most useful. Clear the bowel by the Plombières method and give a course of saline aperients. Keep the patient on a limited diet to relieve the strain on the eliminative organs. Give him copious draughts of fluid to flush the intestines, kidneys and skin.

Encourage free action of the skin by hot baths or hot packs.

Induce him to take exercise in the open air to keep his metabolic processes active.

To carry out these measures it is generally necessary to remove the patient from the worries and distractions of his usual surroundings, and to send him where these can be carried out effectively.

The seaside is out of the question; these patients relapse at once when sent there.

It is for these reasons that Harrogate is so advantageous.
It offers a dry, bracing climate, with ample social amenities, while its natural purifying waters, baths and other facilities make a course of detoxication easy, pleasant and effective.

After detoxication and restoration of the normal functions of the eliminating organs, the removal of the patient's special susceptibility should be taken in hand and further steps taken to prevent relapses.

When possible the patient should be removed from the proximity of his special poison.

When the cause is obvious this is generally easy, except in a trade which may be the only one at which a man can make a living.

When the cause is mysterious this is more difficult.

A patient may have to change completely his diet, his clothes, his life habits, his house, and even his locality before he obtains freedom from relapses.

It is in these mysterious cases that some method of desensitization may be useful.

Natural methods of desensitization are the most practicable and successful.

Duke, in his well-known book on "Allergy," quotes the experience of several American physicians, who relate that workers with "poison ivy" render themselves immune from the dermatitis which it causes by chewing the leaves of the plants.

Personally, when I meet with cases of plant dermatitis such as that due to chrysanthemums, I advise the patients to eat salads of the leaves. Similar steps can be taken with other substances.

The patient can also be habituated to external irritants by gradual exposure to them.

An immunity to sunlight can be obtained by gradual exposure of limited areas as is done in a "sun-bath" treatment.

In food cases the patient can be made immune by habituating him to small but increasing doses of the food to which he is susceptible, on the same principle that anaphylactic shock can be prevented by 
the slow introduction of the anaphylactic substance.

\section{SUMMARY.}

(I) In eczema there is a special susceptibility to external or to absorbed substances.

(2) That this susceptibility may be congenital or acquired.

(3) That the susceptibility is of a similar kind, but not of such frequent occurrence as the allergy present in cases of asthma, hay-fever and urticaria.

(4) That there is a good deal of clinical evidence which indicales that this susceptibility is apt to be developed in those whose metabolic and eliminative functions are defective.

(5) That the best treatment is :-

(a) The removal of the patient from his usual surroundings and particularly from his special poison.

(b) A change in his food, habits and occupation, and, finally,

(c) A course of detoxication and desensitization.

\section{REFERENCE.}

(1) "The Blood-vessels of the Human Skin and their Responses," Sir Thomas Lewis.

\section{BRONCHIECTASIS.}

I POST-(IRADIATE LECTCRE DELIVERED AT YORK.

(Under the auspices of the Fellowship of Medicinc.)

\section{$B y$ L. S. T. BURRELL,}

M.D., F.R.C.P.,

Pliysician to the Royal Free Hospital, and to thi

Brompton Hosfital for Consumption and Diseases of the Chest.

IN a severe case of bronchiectasis, when many of the bronchi have large saccular dilatations, the diagnosis is quite simple. The patient coughs up a quantity of sputum which may be extremely offensive, and which contains pyogenic organisms but no tubercle bacilli. Physical signs vary according to whether the bronchial tubes contain sputum or have been emptied. The bases of the lungs are usually affected and not the apices, as in tuberculosis, and there is almost always considerable clubbing of the fingers.

In less severe cases the diagnosis is often a matter of difficulty, and in some very slight cases it is possible to arrive at a correct diagnosis only after injecting lipiodol into the bronchi and making an X-ray examination.

There is one form of bronchiectasis, described by certain French physicians as the forme sèche hemoptoïque in which the patient suffers from periodic attacks of hæmoptysis, but between the attacks remains quite fit and free from symptoms.

The most common error is to confuse bronchiectasis with pulmonary tuberculosis. In every sanatorium it is common to find a bronchiectasis patient who has been wrongly diagnosed.

This is a serious mistake, not only because the patient is not having the proper treatment for bronchiectasis, but because he is unnecessarily exposed to the restrictions and expense of a consumptive's life. There are three important facts to remember in making a diagnosis :-

(I) Although basal tuberculosis of lung does occur, it is uncommon. If therefore signs are found at the base, the case is unlikely to be one of tuberculosis.

(2) In the early stage of pulmonary tuberculosis, when the spitum first appears, tubercle bacilli may not be found. When, however, the sputum becomes purulent tubercle bacilli are practically always present if the case is one of tuberculosis. Any case of a few months standing with purulent sputum, but without tubercle bacilli, is probably not one of tuberculosis. It is important to bear this in mind, as it is not generally appreciated and one often hears it said that failure to find tubercle bacilli means nothing. Sir James Kingston Fowler collected I86 cases of pulmonary tuberculosis, and of these tubercle bacilli were found in the sputum at the first examination in I 67.

(3) Clubbing of the fingers is almost 